\title{
DIBATTITO FINALE
}

\author{
PIETRO PETRAROIA
}

Aggiungo per cominciare due piccole informazioni, prendendo spunto dalla relazione della professoressa Dubini. Forse non tutti sono al corrente del fatto che Regione Lombardia possiede un archivio di etnografia e storia sociale, forse il più grande d'Europa, di cui non conosco le prospettive di valorizzazione, ma del quale vale la pena di occuparsi. Si può dare consapevolezza dei beni culturali immateriali, e garantirla nel tempo, soltanto se sussiste qualche modalità di registrazione: con questo si pone il problema della gestione dei supporti, dell'archiviazione elettronica e così via, come stamattina si è ricordato. Passo ora alla seconda informazione. Il 20 novembre scorso, in un convegno su temi del restauro che si è tenuto presso l'Archivio di Stato di Roma (alla Sapienza, la sede antica dell'Università), a proposito dell'attuale evoluzione dell'Atto Unico Europeo, ho proposto di sancire che la tradizione italiana del restauro sia riconosciuta come bene culturale immateriale, atteso che molte delle cose di cui abbiamo parlato stamattina sono in realtà totalmente convergenti in una serie di spunti di base ben diversi da quelli della maggioranza di tutti gli altri paesi del mondo, oltre che dell'Europa. E ciò a partire dalla concezione del rispetto per il raccordo tra "originale", "autentico" e "autografo" di cui abbiamo trattato anche a seguito dell'intervento del professor Beretta.

Desideravo anche chiedere se e come è stato ricostruito il raccordo tra la Costituzione di Weimar e quella italiana nell'affermazione della finalità sociale della proprietà privata: credo che la Costituzione italiana, in questo, sia un'eccezione, come era innovativo l'approccio di quella di Weimar. Trovo una singolare coerenza tra questa impostazione, che pone una sorta di obbligo a priori per la proprietà privata di agire nell'interesse generale, nel quale collocare la visione tradizionale "cosificata" della tutela, e una visione legata soprattutto alla cittadinanza attiva delle comunità nel prendersi cura della propria memoria. Se si rimane nell'ambito della tradizione del diritto romano, e quindi della 
protezione della proprietà privata e dei suoi limiti, non si arriva ad avere un quadro di riferimento sufficientemente significativo, per esempio per quanto riguarda i beni immateriali che non si "afferrano", non passano dalla contabilità, non vengono iscritti negli assi ereditari e quindi rischiano di sfuggire a un approccio legato soltanto al diritto civile. Chiedo inoltre che relazione c'è tra l'editto Pacca e la cultura che emerge dal codice napoleonico. Roma era stata saccheggiata dai francesi, tutta la sagrestia di San Pietro è stata dispersa (alcuni elementi sono al Louvre), tuttavia è impressionante l'impronta che il codice napoleonico sembra avere dato alla redazione dell'editto Pacca. Desidero inoltre sottolineare che è all'esame della Commissione Industria del Senato (proposta Lanzillotta-Marcucci, quest'ultimo Presidente della Commissione Cultura del Senato) un provvedimento che concede l'esportabilità dei beni culturali quando il loro proprietario dichiari con una propria valutazione un valore del bene che intende trasferire all'estero inferiore a 150.000 euro; secondo un più recente emendamento il limite dovrebbe essere invece di 13.500 euro, quindi drasticamente inferiore. Si affermerebbe quindi il principio per cui il valore del patrimonio culturale sarebbe legato a una valutazione commerciale, neanche economica. Tale provvedimento è per me inconcepibile, ma forse è perfettamente accettabile per altri, ad esempio per il Presidente dell'Accademia di Brera con cui ne parlavo stamattina. Si tratta di una scelta politica nata all'interno di una Commissione Europea che è all'origine della promulgazione del regolamento e della direttiva posta in appendice al codice dei beni culturali e del paesaggio: in quella sede, in cui rappresentavo l'Italia, mi ero espresso decisamente in senso contrario, come avevano fatto i francesi e i greci, mentre i britannici erano fortemente orientati a definire il patrimonio culturale e la sua esportabilità o non esportabilità in rapporto al valore commerciale. Questo forse dovremmo ricordarcelo in una giornata come quella di oggi che lega il problema dell'identificazione del bene culturale a etica ed economia. Un altro spunto che vorrei offrire, e mi riferisco alla relazione della professoressa Dubini, è invece legato al tema dell'art bonus, istituito probabilmente come scelta di mediazione, ma che, agli effetti finali, è tale da dovere essere necessariamente superato: facilita infatti l'intervento soltanto ai grandi operatori economici, mentre ai piccoli dà pochissimi vantaggi. Inoltre ne sono beneficiari soltanto i beni di appartenenza pubblica, distinzione che contraddice la visione unitaria dei criteri con cui identifichiamo il patrimonio culturale. Siamo, per concludere, a un 
passaggio epocale, perché c'è chi vuole identificare i beni culturali in rapporto al valore commerciale e c'è un art bonus che identifica i "veri" beni culturali da proteggere solo se sono di proprietà pubblica. Io trovo che questo sia assurdo e soprattutto non è mai stato fornito un dato economico essenziale (benché richiesto anche da Italia Nostra, di cui sono vicepresidente): quanto cioè "ci rimetterebbe" lo Stato a seguito della possibilità di applicare l'art bonus anche ai beni di proprietà privata. Se questo allargamento fosse attuato alcune pratiche, come quelle di manutenzione preventiva e programmata, potrebbero essere estese alla generalità del patrimonio, creerebbero una cultura, un'attività imprenditoriale dedicata. Infine volevo informarvi del fatto che, negli ultimi mesi in cui lavoravo in Regione prima del pensionamento, sono stato incaricato di dare avvio a uno studio, completato e attualmente in sviluppo, che porterà a breve all'emanazione da parte di Regione Lombardia di un di atto d'indirizzo su sponsorizzazioni e crowdfunding gestiti dalla Pubblica Amministrazione, tenendo conto di opportunità che la normativa italiana, sorprendentemente, dà in misura più ampia rispetto ad altre europee cercando di navigare tra gli scogli del codice degli appalti, che però in questi giorni è in corso di riforma. Anche su questo tema sarebbe interessante, per chi lo volesse in futuro, fare qualche osservazione di approfondimento sui sistemi del finanziamento complementare (rispetto a quelli tradizionali) a favore del patrimonio culturale, in particolare di appartenenza pubblica.

\section{$* * *$ \\ GIANNANTONIO SACCHI LANDRIANI}

La mia domanda è rivolta a Lunghini: mi pare che avesse affermato, accreditando la frase a Ruskin, che il Rinascimento vede in un certo senso la scienza mortificare l'arte, creando poi un'antitesi tra l'una e l'altra: secondo me è una frase ad effetto che richiederebbe, quantomeno, un'analisi abbastanza approfondita, perché è vera per certi versi, ma falsa per altri.

\section{AMEDEO BELLINI}

Ho due brevissime osservazioni sulla relazione che riguardava gli aspetti giuridici della tutela. Si è fatto cenno a una relazione fra la sua nascita istituzionale con il "patriottismo" tra virgolette. Suggerirei di 
tralasciare le virgolette, dal momento che la conservazione dei monumenti come atto di patriottismo è concetto affermato in documenti ufficiali e ha avuto grandissimo rilievo nella formazione delle tesi sul restauro, in particolare di quello architettonico. L'altra osservazione: giustamente si è detto delle leggi del '39 che sono nate in epoca fascista, ma è bene sottolineare che non sono leggi fasciste: Bottai, quando le vara, le vuole superare proprio per quel motivo, e anche questo si trova in documenti ufficiali. Bottai, le fa approvare ma si rende conto che si fondano su di una concezione tipicamente liberale: fissare diritti e doveri del privato e dello Stato sul piano giuridico e in parte economico, lasciando al dibattito culturale la definizione dei modi di intervento. Una frattura fra azione e pensiero in un'attività, quella della tutela, di alto significato etico (e patriottico) non poteva sfuggire alle scelte dello Stato, al quale competevano, appunto, le scelte di natura etica. Egli assume iniziative forti, di elaborazione teorica, di natura propagandisti$\mathrm{ca}, \mathrm{di}$ carattere editoriale che si possono sintetizzare nella volontà di pervenire alla coincidenza tra una teoria del restauro e una legge di tutela, da inserire anche nella carta della scuola per le inevitabili implicazioni didattiche.

Si indica l'arte come "non sostenibile", come un'attività che dal punto di vista economico non sostiene sé stessa. L'affermazione suggerisce un tema: la fruizione di un bene culturale deve essere pagata o no? Qualcuno ha scritto (Manacorda in una lettera a "Repubblica"), replicando ad affermazioni contrastanti, che si tratta di due nobili aspirazioni fra le quali è difficile scegliere. A mio parere non è così; si dice "gratis", ma questa è una falsa parola. Penso che gli economisti siano d'accordo sul fatto che "gratis" non esiste, che tutto ha un costo, anche la produzione e la conservazione di un bene culturale. Si tratta di stabilire chi e in che misura deve pagare. È una scelta che riguarda molti campi, per esempio i servizi pubblici di trasporto: quanto paga la comunità e quanto il singolo fruitore, partendo dal presupposto che anche coloro che non fruiscono direttamente del servizio ne hanno indirettamente un beneficio? C'è infatti un interesse nazionale all'esistenza di un sistema di trasporto efficiente. Vorrei sapere se dal punto di vista della gestione dei beni culturali questa questione sia stata affrontata. Nel settore dei trasporti si è modificato nel tempo il rapporto tra costo a carico del fruitore e costo pubblico, dopo un dibattito che ha tenuto conto delle quantità di denaro in gioco e di valutazioni politiche. Una ulteriore domanda la rivolgo a Giorgio Lunghini, domanda che forse va oltre i 
contenuti di questo convegno e quindi andrà rinviata ad altro momento: l'economia keynesiana, vista in prospettiva attuale, non rischia di giustificare una società dei consumi a sviluppo illimitato in cui produzione, consumo e aumento dei consumi per ottenere un aumento della produzione finiscono per perdere di vista l'obiettivo di soddisfare $\mathrm{i}$ bisogni divenendo invece fine a se stessi? Questa è una domanda di fondo, connessa al ruolo che potrebbe avere la fruizione dei beni culturali immateriali, in quanto limite alla fruizione dei beni di consumo.

\section{SILVIO BERETTA}

Ho una curiosità con riferimento alla relazione della collega Dubini. Ricordo di avere letto qualche tempo fa di studi, condotti soprattutto nell'Università Bocconi, in materia di sostenibilità, onerosità e redditività delle manifestazioni artistiche. Le conclusioni che venivano tratte variavano a seconda anche di circostanze temporali. In particolare, la manifestazione che durava molto rendeva molto, o comunque si sosteneva: era quindi opportuno protrarre una determinata esposizione di opere d'arte, mentre la redditività diminuiva e l'onerosità aumentava mano a mano che i tempi di esposizione si accorciavano. Dall'attività di ricerca e di verifica di quella Università sembrava quindi risultare una sorta di "sistematica" utile a valutare costi e benefici delle manifestazioni artistiche. Mi accodo poi a due osservazioni del professor Petraroia. La prima verte sulla questione dell'art bonus. Ho visitato la settimana scorsa, a New York, la Morgan Library e la Whitney Collection. In entrambe, l'elenco dei donatori si misurava in metri quadrati, ma si trattava esclusivamente di contributi privati: nemmeno un dollaro era pubblico, compresa l'incastellatura che Renzo Piano aveva costruito per entrambi i musei. Il tutto, naturalmente, era fiscalmente agevolato, il che spiega entità e numerosità dei contributi raccolti. La seconda osservazione si riferisce all'emendamento Marcucci-Lanzillotta, del quale la stampa si è occupata e sul quale chiedo un'opinione dei relatori.

\section{ANTONIO PADOA SCHIOPPA}

Mi permetto di proporre un codicillo all'osservazione che Lunghini ha fatto citando Benjamin, secondo il quale "la riproduzione 
di un'opera d'arte ne fa perdere l'autenticità". Se fossi un legislatore (certamente non lo sarò mai) vieterei di deturpare alcune delle più grandi opere d'arte attraverso la pubblicità sui giornali. Quando vedo Monna Lisa con i baffi ritengo trattarsi di un reato, perché viene banalizzato uno dei vertici dell'arte mondiale e questo non dovrebbe essere permesso. Posso ammettere Einstein che tira fuori la lingua, anche se si tratta di una esagerazione, ma con l'arte non si scherza. Se si può discutere sul fatto che la riproducibilità può banalizzare, quello su cui non si può discutere è il fatto che i capolavori non si possono deturpare. Gli Stati Uniti sono certamente un modello per quanto riguarda la tutela delle loro peculiarità; chi di noi vi è stato ha constatato che, anche a un monumento che da noi passerebbe totalmente inosservato, viene apposta una targa del tipo "Monumento storico n. 565 del Vermont", e magari si tratta di una fontanella. Gli Stati Uniti hanno una storia molto recente, però si stanno muovendo in questa direzione; noi, da questo punto di vista, dovremmo invece coprire l'intero territorio. Quanto al fatto che citava ora Silvio Beretta, mi pare di poter dire che questo è legato in modo molto evidente alle circostanze del vantaggio fiscale: i privati infatti, salvo pochissime eccezioni sempre più rare (e in questo senso l'Italia ha fatto passi indietro giganteschi rispetto al passato) non contribuiscono se non ne ricavano vantaggi. Negli Stati Uniti questi vantaggi sono evidentissimi sia sotto il profilo successorio sia anche per quello che riguarda gli sgravi. Questa politica mi pare legittima; tuttavia oggi si va diffondendo una sorta di ideologia privatistica, mentre certe tutele, non solo dell'arte ma anche del territorio, richiedono interventi pubblici, denaro pubblico. Keynes lo sapeva bene. Certamente l'Europa qualcosa sta facendo, anche se con procedure troppo complicate e, come spesso accade, se c'è un incentivo europeo anche l'Italia si muove. Quindi il tema è fondamentale, essendo l'Italia il paese del mondo che ha il patrimonio culturale di gran lunga più ricco, con gli enormi doveri e i potenziali vantaggi che ne conseguono. Se penso a quello che si è fatto per la cultura nei primi anni della Regione Lombardia e a quello che si sta invece facendo ora, almeno nei campi che conosco, i passi indietro sono stati enormi. Un solo esempio. Nei primi anni si sono tutelate le autonomie locali facendo una ricognizione con microfilm di tutti gli statuti dei paesi e delle città lombarde, che sono più di 2.000. Oggi, invece, questo tipo di ricerca sarebbe inattuabile. Si comincia un progetto ma poi lo si interrompe... Potrei fare molti altri esempi ma mi fermo qui. 


\section{ELISABETTA FUSAR POLI}

Inizio con il rispondere all'osservazione relativa alla connotazione fascista delle leggi del '39. Mi riferivo non tanto alle norme in sé quanto al contesto. Volevo solo aggiungere che non è un caso che proprio in quel periodo si sia convogliata l'attenzione su questo tipo di problematica, proprio perché il patrimonio culturale era concepito come strumento propagandistico, tra $\mathrm{i}$ tanti, e di rafforzamento del prestigio a livello internazionale, di affermazione della preminenza dello Stato italiano sugli altri a livello europeo e mondiale: quindi uno strumento marcatamente politico nelle mani del regime. La normativa è stata voluta anche per questo. Il fatto è che, come per tante altre disposizioni che hanno visto la luce nel periodo fascista, non posso fare altro che confermare che ci ha lavorato un' intellighentia trasversale, non solo fascista. I resoconti dei dibattiti lo attestano e attestano come i loro esiti prescindessero dagli schieramenti politici, ma che sono stati frutto di punti fermi raggiunti attraverso uno scambio di idee altissimo, il cui spessore intellettuale è veramente impressionante e non ha nulla a che vedere con la connotazione ideologica che si può sospettare a una lettura superficiale. Circa la funzione sociale della proprietà, è assolutamente vero che la tematica ha avuto un rilievo anche nel dibattito relativo al patrimonio culturale nazionale, soprattutto dopo la Costituzione, ma anche prima in realtà, perché è un elemento di riflessione che ha matrici anteriori e che riscontriamo nel dibattito intorno a questi temi. Devo però dire che non è tra gli elementi prevalenti per giustificare un'esigenza di tutela: non è un aspetto che assuma, da un punto di vista giuridico, un rilievo particolarmente determinante. Forse non è stato sufficientemente sviluppato e comunque non ha trovato nelle sedi legislative un riscontro significativo; lo si riscontra di più nel parallelo dibattito dottrinale, ma non ha esiti concreti dal punto di vista normativo. È più rilevante la configurazione dell'immaterialità, che viene presa in prestito dalla riflessione intorno alle opere dell'ingegno, quindi del diritto d'autore e di tutto quello che vi è connesso quando l'autore è ancora in vita, mentre l'idea di "patrimonio culturale" si costituisce quando l'autore dell'opera non è più in vita. Questo elemento di discrimine consente di formulare alcune riflessioni molto utili per la costruzione della normativa dalla materia delle opere dell'ingegno a quella del patrimonio culturale. Questo è un dato che sembra avere un riscontro effettivo dal 
punto di vista della riflessione giuridica, più evidente rispetto a quello della funzione sociale della proprietà, che pure è presente.

\section{PAOLA DUBINI}

Primo tema è l'art bonus: non c'è dubbio che si tratti di una forma di incentivo ancora da mettere a punto e sulla quale si sta cominciando a ragionare; anch' io penso che l'idea che vengano esclusi i beni di proprietà privata sia una sciocchezza, perché ci si concentra sulla proprietà, pubblica o privata, creando una serie di problemi e di disparità laddove invece il senso è il sostegno a un'attività con l'obiettivo di ottenere un beneficio di natura pubblica. Non sono completamente sicura che il vantaggio per il donatore favorisca, se non nelle cifre assolute, il grosso investitore, l'impresa piuttosto che il privato. Credo che ci sia una fase di sperimentazione: considerando quanti sono a oggi quelli che effettivamente hanno contribuito, il numero di privati intervenuti con somme inferiori a 1.000 euro non è piccolissimo e se dall'altra parte considero l'elenco dei progetti, ce ne sono molti che hanno riguardato il restauro del singolo quadro, con un valore di 500 euro, che assolutamente non favorisce l'intervento dell'azienda che voglia fare un'azione di mecenatismo; quindi secondo me l'art bonus è un esempio di nuova iniziativa sulla quale si sta concentrando interesse e sulla quale ci sarà una quantità enorme di lavoro da fare. Il fatto di avere pubblicato sul sito un progetto non significa minimamente che esso venga finanziato: a oggi più di metà non hanno donazioni e il numero di quelli completamente finanziati si conta sulle dita di due mani; siamo quindi veramente in una fase molto iniziale. È chiaro che dovrà imparare sia chi dona sia chi presenta i progetti. Penso che dobbiamo aspettarci, nel prossimo futuro, una crescita molto rilevante del numero di progetti proposti a fronte di un sistema informativo che non permette neanche di effettuare un minimo di selezione a priori; quindi c'è veramente molto da fare e sono assolutamente d'accordo che non ha senso che non ci siano progetti presentati da privati. Riguardo alla sostenibilità, evidentemente non sono stata chiara. Quando dico che l'attività non è sostenibile mi riferisco a quella di tutela: se noi la consideriamo nel senso stretto del termine, essa di per sé non genera reddito. Per esempio l'attività delle biblioteche è strutturalmente in perdita. Questo non significa, naturalmente, che non si possa immaginare di gestire un bene culturale con una logica privati- 
stica e questo spiega come mai si abbiano così tanti festival, luoghi della cultura che affittano spazi e addirittura ci siano anche forme giuridiche che vanno nella direzione di mescolare le finalità no profit con quelle di tipo economico. Riguardo a questo, confermo quanto diceva il professor Petraroia, cioè che abbiamo effettivamente una normativa attenta a questo fenomeno, sia sulle forme miste (penso alle cosiddette B Corp su cui l'Italia è molto avanzata), sia, incredibilmente, sul crowdfunding. Quando dicevo che non c'è sostenibilità mi riferivo specificatamente all'attività di tutela; secondariamente, è chiaro che, soprattutto in una logica di autonomia, la scelta del prezzo eventuale o della gratuità da parte del singolo soggetto diventa una delle leve strategiche. Così come ci sono delle imprese che possono decidere di svolgere delle attività in perdita e quindi immagineranno di avere altre attività che le sovvenzionano, tanto più questo vale per iniziative che hanno una funzione in toto o in parte di natura pubblica, collettiva.

\section{GIORGIO LUNGHINI}

Prima osservazione: Keynes non si sarebbe mai sognato di rifiutare contributi privati per la tutela, ma la sua critica principale è rivolta a chi dice che lo Stato non può permetterselo perché sarebbe uno spreco; sono due cose completamente diverse. Circa Benjamin e l'autenticità, la parola più importante in quel passo è quella dell' “aura" dell'opera d'arte, che è estremamente evocativa di un rapporto diretto e non mercantile. L'altra parola che conta è "culturale". In un certo senso c'è qualcosa in comune tra Benjamin e Ruskin: se mi sentissero, cosa che è impossibile, rifiuterebbero questo accostamento; però questa dimensione non mercantile del rapporto con l'opera d'arte è presente in tutti e due. Ruskin lo trova in particolare nel Medioevo e la questione del Rinascimento credo sia solamente un espediente metodico: denigrare il Rinascimento per consentire l'apologia del Medioevo. La questione di Keynes come possibile promotore di un sistema consumista? No, nella maniera più assoluta. Lui amava la bella vita, ma amava anche il buon gusto, la raffinatezza, la modestia. Il suo filosofo di riferimento era Moore, il cui motto era "fate il bene", e comunque la sua politica economica si indirizzava su tre interventi possibili. Dapprima una forte redistribuzione del reddito dai ricchi ai poveri, cosa predicata dalla Costituzione italiana ma mai realizzata, perché basterebbe rendere pro- 
gressiva l'imposizione per ottenere il primo dei tre risultati auspicati da Keynes. Il secondo, l'avevo già nominato prima, è l'eutanasia del rentier, il quale fondamentalmente è un tale che vive del diritto di proprietà, non più della proprietà della terra come era in epoca precapitalistica, ma dei mezzi finanziari; la sua condanna del consumismo è esplicita quando dice che chi desidera il denaro di per sé soffre di una malattia la cui cura andrebbe delegata a uno psichiatra. Quindi Keynes non ha affatto una visione consumistica. La terza linea di intervento che Keynes suggerisce è una non piccola socializzazione degli investimenti, con il che non chiede che lo Stato si sostituisca ai privati, ma che faccia ciò che i privati non fanno e noi vediamo benissimo nel nostro Paese che ci sono tante cose desiderabili che i privati non trovano conveniente fare (quindi non gli si può rimproverare di non farla), ma in quel caso dobbiamo chiedere allo Stato di intervenire.

\section{AMEDEO BELLINI}

Sarò brevissimo: voglio rispondere al quesito posto da Sacchi Landriani, dissentendo in parte da Giorgio Lunghini: in estrema sintesi Ruskin, nella sua visione romantica dell'arte e nella convinzione che questa non fosse altro che la "trascrizione" del bello naturale, non ammetteva alcuna possibilità di una sua razionalizzazione, del suo uso per un'espressione concettuale, come è avvenuto nel Rinascimento. Da qui l'avversione soprattutto verso Raffaello, il sostegno al movimento a lui contemporaneo dei "preraffaelliti" e la condanna severa del manierismo, che gli appariva imitazione di una falsità. 